\title{
Differences in 23S ribosomal RNA mutations between wild-type and mutant macrolide-resistant Chlamydia trachomatis isolates
}

\author{
YONG JIANG ${ }^{1}$, HUI ZHU ${ }^{1}$, LI-NA YANG ${ }^{1}$, YUAN-JUN LIU ${ }^{2}$, \\ SHU-PING HOU ${ }^{2}$, MAN-LI QI ${ }^{2}$ and QUAN-ZHONG LIU ${ }^{2}$ \\ ${ }^{1}$ Department of Dermatology, The Second Hospital of Tianjin Medical University; \\ ${ }^{2}$ Department of Dermatology, General Hospital of Tianjin Medical University, Heping, Tianjin 300052, P.R. China
}

Received July 26, 2014; Accepted April 16, 2015

DOI: $10.3892 /$ etm.2015.2595

\begin{abstract}
The aim of the present study was to determine the in vitro susceptibility of wild-type and mutant clinical isolates of Chlamydia $(C$.) trachomatis strains to erythromycin, azithromycin and josamycin, and to identify the resistance-conferring $23 \mathrm{~S}$ ribosomal (r)RNA mutations in the isolates. The wild-type resistant isolates were defined as those with minimum inhibitory concentration values above the tissue concentration of the antibiotic in the urogenital system. Furthermore, all resistant $C$. trachomatis isolates were exposed to sub-inhibitory concentrations of macrolides, and 13 resistant mutants were selected following serial passages. Among the 8 wild-type isolates that were resistant to erythromycin, 3 isolates had a mutation at T2611C in the 23S rRNA gene while the others did not show any 23S rRNA mutations. The selected mutant isolates showed a 4 - to 16-fold reduction in in vitro sensitivities. With regard to the mutant strains, the T2611C mutation was found in 10 isolates, A2057G mutation in 6 isolates, and A2059G mutation in 1 isolate. Thus, the macrolide-resistant isolates of the wild-type strain had different mutations from those selected by exposure to sub-inhibitory concentrations of macrolides. Also, since 23S rRNA mutations were not identified in certain isolates, it was considered that other molecular mechanisms may also be responsible for the macrolide resistance of $C$. trachomatis.
\end{abstract}

\section{Introduction}

Chlamydia $(C$.) trachomatis is an obligate intracellular human pathogen that leads to numerous inflammatory conditions in

Correspondence to: Professor Quan-Zhong Liu, Department of Dermatology, General Hospital of Tianjin Medical University, 154 Anshan Street, Heping, Tianjin 300052, P.R. China

E-mail: liuquanzhong@medmail.com.cn

Key words: Chlamydia trachomatis, macrolide, 23S rRNA, point mutation the urogenital tract. It is the most common bacterial species that causes sexually transmitted infections (1). Despite appropriate drug therapy, chlamydial infections are highly likely to recur. The majority of clinical failures are due to reinfection or relapse following phenotype deviation of the bacteria to persistent, non-replicating types that are antibiotic resistant but can revert to the typical reticulate body phenotype once treatment is complete $(2,3)$. Currently, the recommended first-line therapeutic regimen for chlamydial infections is the administration of tetracyclines and macrolides, which impede bacterial translation by binding to $30 \mathrm{~S}$ and $50 \mathrm{~S}$ ribosomal subunits, respectively (4). Clinical isolates from patients with recurrent $C$. trachomatis infection have been shown to have resistance against macrolides (5-7).

Under laboratory conditions, the substitution of a single base in ribosomal (r)RNA has been shown to result in macrolide resistance. This form of resistance was first identified in yeast, where the mitochondrial operon was mutated at position A2058 of the large-subunit rRNA (8). Subsequently, similar phenotypes were obtained in Escherichia $(E$.) coli by the expression of mutant rRNA alleles from multiple-copy plasmids (9). Several years later, further reports of rRNA mutations that conferred macrolide resistance to clinical pathogens began to appear in the literature (9-11). Mutations at positions 2057, 2058, 2059 and 2611 (E. coli numbering) in the peptidyl transferase region of $23 \mathrm{~S}$ rRNA are considered to be important in the development of drug resistance against macrolides (12). Reports of clinical failures linked to true genotypic resistance due to chromosomal mutations are rare.

Mutations in the 23S rRNA gene were initially reported in macrolide-resistant $C$. trachomatis strains in 2004 (13); 4 clinical isolates were observed to be resistant to all macrolides and were found to harbor A2058C and T2611C mutations (E. coli numbering). A more recent study, however, identified no mutations in the $23 \mathrm{~S}$ rRNA genes of resistant mutants that were selected following enrichment by serial passage in the presence of sub-inhibitory concentrations of azithromycin (14).

The objective of the present study was to investigate mutations in the 23S rRNA gene of macrolide-resistant isolates of wild-type $C$. trachomatis obtained from clinical samples and mutant strains selected using sub-inhibitory concentrations of the macrolides. 
Table I. Nucleotide sequences of the primers used for reverse transcription-polymerase chain reaction.

\begin{tabular}{lllr}
\hline Gene & Primer & \multicolumn{1}{c}{ Sequence $\left(5^{\prime} \rightarrow 3^{\prime}\right)$} & Position $^{\mathrm{a}}$ \\
\hline 23S rRNA & RR-forward & AAGTTCCGACCTGCACGAATGG & 2004 \\
& RR-reverse & TCCATTCCGGTCCTCTCGTAC & 2728 \\
\hline
\end{tabular}

a Position for the first base of the primer sequence at GenBank (GI: 5042363).

\section{Materials and methods}

Bacterial strains and cells. C. trachomatis isolates were obtained from patients who attended the Tianjin Institute of Venerology (Tianjin, China) during 2005-2008. Each patient was sampled for only 1 isolate. The reference strain C. trachomatis E-UW-5/Cx was obtained from the Chlamydia Research Center of Maryland University (Baltimore, MD, USA). McCoy cells (Institute of Dermatology, Chinese Academy of Medical Sciences, Nanjing, China) were grown in culture medium (minimal essential medium supplemented with $10 \%$ fetal bovine serum and $2 \mathrm{mM} \mathrm{L}$-glutamine) and were incubated at $37^{\circ} \mathrm{C}$ in a $5 \% \mathrm{CO}_{2}$ atmosphere.

Antibiotics. The antimicrobial agents examined were erythromycin (Sigma-Aldrich, Munich, Germany), azithromycin and josamycin (National Institute for the Control of Pharmaceutical and Biological Products, Beijing, China).

Determination of the minimum inhibitory concentration (MIC). Cultured McCoy cells were seeded into 96-well plastic plates and incubated for $24 \mathrm{~h}$ at $37^{\circ} \mathrm{C}$ in a $5 \% \mathrm{CO}_{2}$ atmosphere; they were then inoculated with $4 \times 10^{4}$ inclusion-forming units of the bacterial strains per milliliter of the medium. The plates were centrifuged at $1,200 \mathrm{x}$ g at $37^{\circ} \mathrm{C}$ for $1 \mathrm{~h}$ and were incubated for $2 \mathrm{~h}$ at $37^{\circ} \mathrm{C}$ in a $5 \% \mathrm{CO}_{2}$ atmosphere. Then, the cell monolayers were overlaid with a 2-fold dilution series of antibiotics in growth medium (culture medium supplemented with cycloheximide at a concentration of $1 \mu \mathrm{g} / \mathrm{ml})$. The macrolide concentrations ranged from 0.25 to $2 \mu \mathrm{g} / \mathrm{ml}$ for erythromycin, from 0.063 to $1 \mu \mathrm{g} / \mathrm{ml}$ for azithromycin and from 0.02 to $0.16 \mu \mathrm{g} / \mathrm{ml}$ for josamycin. The plates were incubated for $48 \mathrm{~h}$ at $37^{\circ} \mathrm{C}$ in $5 \% \mathrm{CO}_{2}$. The cell monolayers were then fixed in methanol, stained with iodine for chlamydial inclusions and observed under an inverted microscope (YYJ-200E; Shanghai Instrument Circular Optical Instrument Co., Ltd., Shanghai, China) at a magnification of $\mathrm{x} 400$. The MIC was defined as the lowest antibiotic concentration at which no inclusions were observed (15-17). The wild-type isolates were defined as resistant to an antibiotic when the MIC value of the antibiotic was greater that its tissue concentration in the urogenital system (18).

Selection of resistant mutants. Macrolide-resistant mutants of $C$. trachomatis were selected by successive passages of the strains in the presence of sub-inhibitory concentrations of erythromycin, azithromycin and josamycin. Firstly, confluent McCoy cell monolayers in 12-well plates were inoculated with $\sim 10^{8}$ inclusion-forming units of the isolated C. trachomatis strains. Growth medium supplemented with $0.5,0.5$ and
$0.04 \mu \mathrm{g} / \mathrm{ml}$ of erythromycin, azithromycin and josamycin, respectively, were added $2 \mathrm{~h}$ later. The infected cells were incubated at $37^{\circ} \mathrm{C}$ in $5 \% \mathrm{CO}_{2}$ for $48 \mathrm{~h}$, and inclusions were observed. The passages were repeated with the same macrolide concentrations until a highly infectious inoculum was obtained, and the MICs for these selected strains were determined. The investigations were conducted in duplicate. Strains for which MICs showed a $\geq 4$-fold increase were considered to be resistant mutants (19).

Amplification of 23S RNA. Total RNA was isolated from infected McCoy cells $48 \mathrm{~h}$ after infection using TRIzol reagent (Tiangen Biotech Co., Ltd., Beijing, China) according to the manufacturer's instructions. RNA was extracted with chloroform, precipitated with isopropanol and rinsed with ethanol $(18,19)$. DNase-treated RNA was examined by polymerase chain reaction (PCR) to ensure complete DNA removal. Control RNA isolated from uninfected McCoy cells and C.trachomatis E-UW-5/Cx were extracted using the same protocol.

Complementary (c)DNA was synthesized from 3-4 $\mu \mathrm{g}$ RNA using Avian Myeloblastosis Virus Reverse Transcriptase (Bioflux, Tokyo, Japan) and specific primers (13) (Table I; Invitrogen, Shanghai, China). PCR amplification of the cDNA of $23 \mathrm{~S}$ rRNA was conducted using specific forward and reverse primers, whose nucleotide sequences were deduced from highly conserved motifs of $C$. trachomatis serovar L2. The two specific primers flanked a 725-bp DNA fragment from the resistant mutant strains of $C$. trachomatis.

The PCR procedures were performed in a final solution (volume, $50 \mu \mathrm{l}$ ) containing each primer; it was composed of $200 \mathrm{mM}$ deoxynucleoside triphosphates, $3 \mathrm{mM} \mathrm{MgCl}_{2}, 5 \mu \mathrm{l}$ 10X Taq buffer, 2 units Taq polymerase (Takara, Dalian, China) and 100 ng purified cDNA.

The PCR protocol was as follows: Denaturation for $5 \mathrm{~min}$ at $95^{\circ} \mathrm{C} ; 35$ cycles of amplification for $40 \mathrm{sec}$ at $95^{\circ} \mathrm{C}, 40 \mathrm{sec}$ at $60^{\circ} \mathrm{C}$ and $1 \mathrm{~min}$ at $72^{\circ} \mathrm{C}$; and a final extension for $10 \mathrm{~min}$ at $72^{\circ} \mathrm{C}$. Negative controls containing DNA extracted from uninfected McCoy cells were included in each PCR experiment. In addition, DNase-treated RNA was examined by PCR to ensure complete DNA removal.

DNA purification and sequencing. The amplification products of $C$. trachomatis and macrolide-resistant strains were purified and directly sequenced at Invitrogen and Tiangen Co.

Accession numbers of the nucleotide sequences. The nucleotide sequence data for the 23S rRNA sequences have been deposited in GenBank with the GI number: 5042363. 


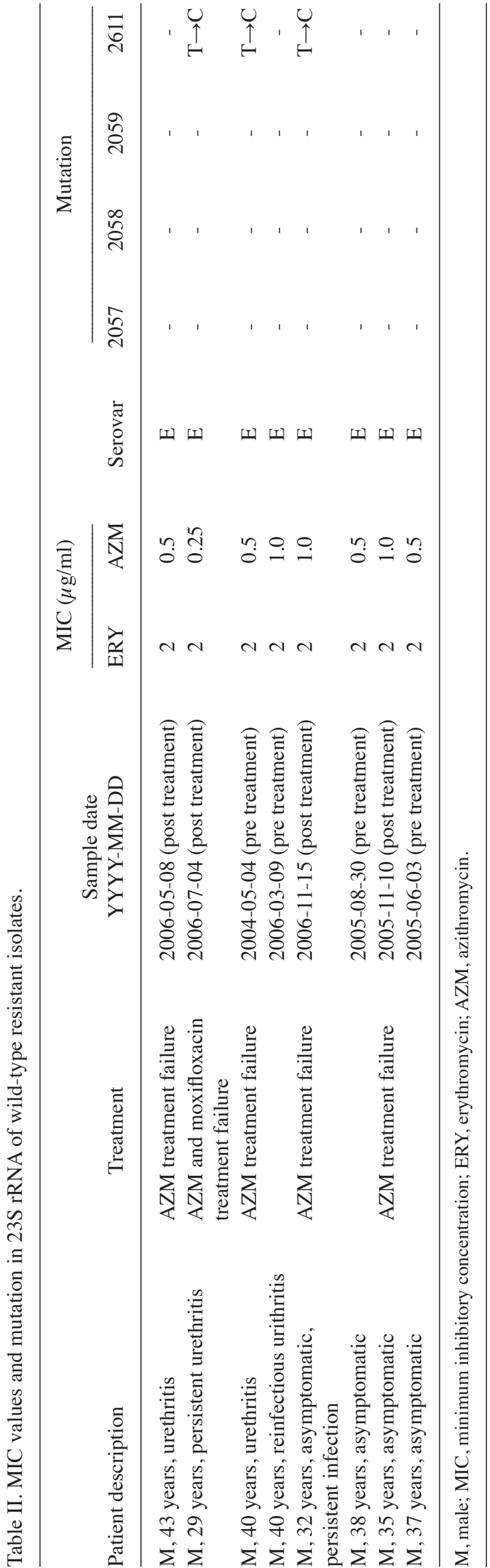

\section{Results}

Selection of wild-type resistant strains. Clinical strains of C. trachomatis were isolated and the in vitro MICs of antibiotics in these strains were determined. The MIC values of erythromycin in the 8 strains of $C$.trachomatis were found to be higher than the concentration of erythromycin in the blood $(1 \mu \mathrm{g} / \mathrm{ml})$, and even higher than the tissue concentration of erythromycin in the urogenital system (Table II). These were considered to be wild-type macrolide-resistant strains of $C$. trachomatis. Furthermore, their sensitivity to azithromycin was lower than it was earlier considered to be (7).

Selection of resistant mutants. In total, there were 13 strains of C. trachomatis that demonstrated resistance to 14-membered (erythromycin), 15-membered (azithromycin) and 16-membered (josamycin) macrolides after 20 passages; therefore, these thirteen strains were selected as resistant mutants (Table III). In the C. trachomatis variants, the azithromycin and erythromycin MICs were 4- to 16-fold higher and the josamycin MICs were 4- to 8-fold higher than the corresponding MICs for wild-type resistant strains.

Amplification of the 23S rRNA gene. The 23S rRNA genes of resistant isolates were amplified. The size of the amplification product was $725 \mathrm{bp}$.

Mutations in the 23S rRNA of wild-type resistant isolates. In the $23 \mathrm{~S}$ rRNA gene of the 8 wild-type resistant isolates, no resistance-associated mutations were found at 2057 (E. coli numbering), 2058 or 2059 and only 3 resistant isolates had the T2611C mutation (Table II). In the case of 2 patients with persistent infection, the isolates had the T2611C mutation.

Mutations in the $23 S$ rRNA of mutant isolates with selective resistance. No mutations were found at 2058 (Table III). A2057G mutations were found in 6 mutant isolates, and T2611C mutations were found in 10 mutant isolates. Two mutant resistant isolates showed A2059G mutations, while 2 of the resistant isolates did not show any mutations in their $23 \mathrm{~S}$ rRNA sequences. The medical records of the patients indicated that those infected with mutant strains did not respond to azithromycin.

\section{Discussion}

In this study, a set of resistant clinical isolates of $C$. trachomatis were differentiated into wild-type and mutant strains and the 23S rRNA mutations in the isolates were identified.

The sensitivity of the wild-type clinical isolates to erythromycin and azithromycin was found to be lower than that reported previously (7). This may explain the high recurrence rate and treatment failure reported for chlamydial infections. In the patients included in the present study, azithromycin treatment was not successful in the case of 8 resistant strains. For 2 patients with persistent infection, the T2611C mutation was found in the isolates. The other wild-type resistant strains had no mutation in the 23S rRNA; it is therefore possible that other molecular mechanisms were responsible for their resis- 


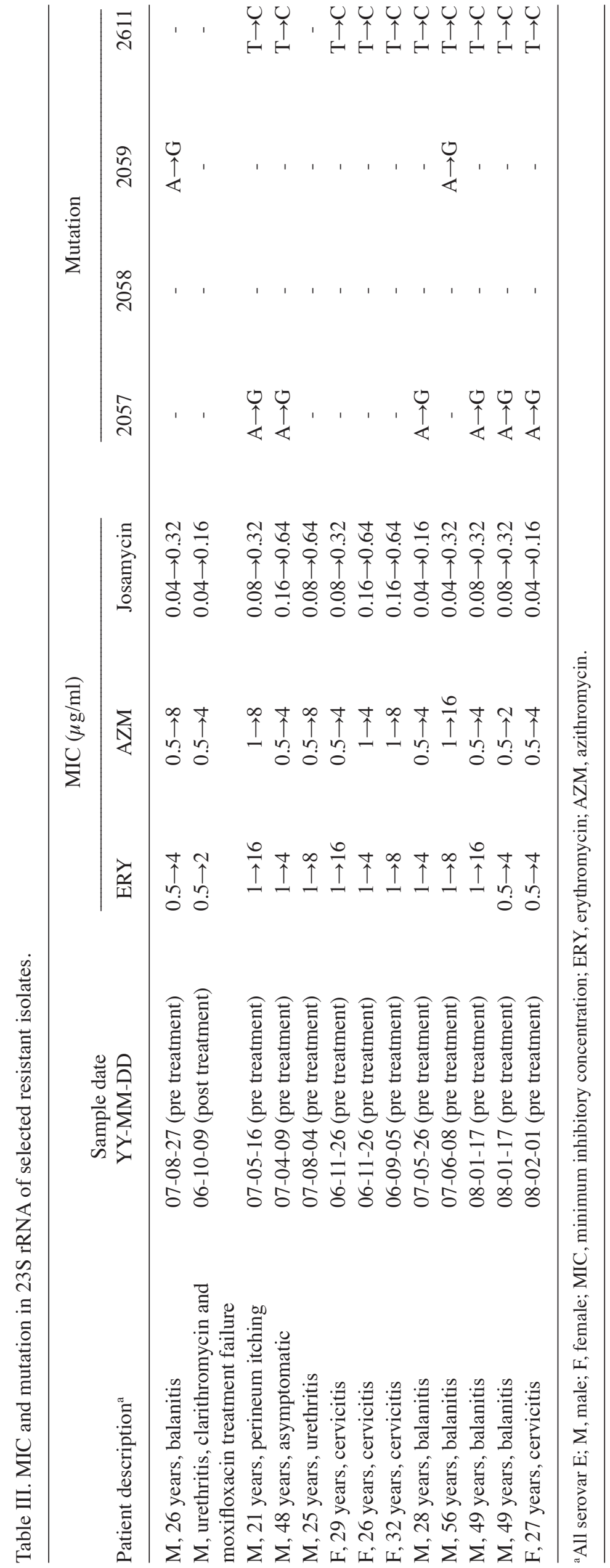


tance. Possible mechanisms underlying the resistance should be investigated in the future.

The 13 mutant resistant isolates showed a 4- to 16 -fold reduction in azithromycin and erythromycin sensitivities and a 4- to 8-fold reduction in josamycin sensitivities compared with the wild-type resistant isolates. The majority of them had a T2611C mutation, which was also found in 2 wild-type resistant strains; therefore, the T2611C mutation may have been responsible for the selective resistance. An A2057G mutation in 6 strains and an A2059G mutation in 2 strains were also found to confer resistance; mutations at these two points were also associated with wild-type resistance. The 2 resistant strains with no $23 \mathrm{~S}$ rRNA mutations require further investigation. Both the wild-type and mutant strains had no A2058 mutations, which reportedly confer the highest levels of resistance (12).

Other mechanisms that confer resistance to C.trachomatis require study in order to understand the resistance of isolates that have no mutation in the peptidyl transferase region of the 23S rRNA gene. To date, there have been no reports of resistance conferred by macrolide inactivation, and the resistance developed by modification of endogenous efflux systems or by drug inactivation remains to be assessed. It is possible that these mechanisms played a role in the resistance of isolates with no 23S rRNA mutations.

To the best of our knowledge, this is the first time that wild-type macrolide-resistant $C$. trachomatis strains have been observed in vitro. Previous studies have identified wild-type strains with selective resistance under antibiotic pressure (12). This also appears to be the first time that A2057G and A2059G mutations in the peptidyl transferase region of the 23S rRNA gene have been found in $C$. trachomatis with selective macrolide resistance. Misyurina et al (13) reported only A2058C and T2611C mutations in macrolide-resistant $C$. trachomatis isolates.

Vester and Douthwaite (12) reported that mutations discovered in clinical strains were also observed in laboratory strains although the reverse was not found to be true. This is likely to be because rRNA mutations leading to drug resistance in a clinical pathogen often only first become apparent once a drug therapy program has failed to eradicate the pathogen. Drug therapies can result in strains containing mutations that confer the highest resistance becoming prevalent. By contrast, rRNA mutations in laboratory strains are intentionally created in order to evaluate drug interaction mechanisms. Under controlled laboratory conditions, it is only possible to create phenotypes with less effective resistance. Such artificially created rRNA mutations may help to delineate the macrolide interaction site on the ribosome. However, unless they segregate with another resistance mechanism, the mutations are unlikely to be observed in clinical isolates. In conclusion, therefore, macrolide-resistant isolates of wild-type $C$. trachomatis are likely to have different mutations from those selected under laboratory conditions.

\section{References}

1. Centers for Disease Control and Prevention; Workowski KA and Berman SA: Sexually transmitted diseases treatment guidelines, 2006. MMWR Recomm Rep 55: 1-94, 2006.

2. Hogan RJ, Mathews SA, Mukhopadhyay S, Summersgill JT and Timms P: Chlamydial persistence: Beyond the biphasic paradigm. Infect Immun 72: 1843-855, 2004.

3. Mpiga $\mathrm{P}$ and Ravaoarinoro M: Chlamydia trachomatis persistence: An update. Microbiol Res 161: 9-19, 2006.

4. Senn L, Hammerschlag MR and Greub G: Therapeutic approaches to Chlamydia infections. Expert Opin Pharmacother 6: 2281-2290, 2005.

5. Somani J, Bhullar VB, Workowski KA, Farshy CE and Black CM: Multiple drug-resistant Chlamydia trachomatis associated with clinical treatment failure. J Infect Dis 181: 1421-1427, 2000.

6. Horner P: The case for further treatment studies of uncomplicated genital Chlamydia trachomatis infection. Sex Transm Infect 82: 340-343, 2006

7. Samra Z, Rosenberg S, Soffer Y and Dan M: In vitro susceptibility of recent clinical isolates of Chlamydia trachomatis to macrolides and tetracyclines. Diagn Microbiol Infect Dis 39: 177-179, 2001.

8. Sor F and Fukuhara H: Identification of two erythromycin resistance mutations in the mitochondrial gene coding for the large ribosomal RNA in yeast. Nucleic Acids Res 10: 6571-6577, 1982.

9. Sigmund CD, Ettayebi M and Morgan EA: Antibiotic resistance mutations in $16 \mathrm{~S}$ and $23 \mathrm{~S}$ ribosomal RNA genes of Escherichia coli. Nucleic Acids Res 12: 4653-4663, 1984.

10. Meier A, Heifets L, Wallace RJ Jr, et al: Molecular mechanisms of clarithromycin resistance in Mycobacterium avium: Observation of multiple $23 \mathrm{~S}$ rDNA mutations in a clonal population. J Infect Dis 174: 354-360, 1996.

11. Scarpellini P, Carrera P, Cavallero A, Cernuschi M, Mezzi G, Testoni PA, Zingale A and Lazzarin A: Direct detection of Helicobacter pylori mutations associated with macrolide resistance in gastric biopsy material taken from human immunodeficiency virus-infected subjects. J Clin Microbiol 40: 2234-2237, 2002.

12. Vester B and Douthwaite S: Macrolide resistance conferred by base substitutions in $23 \mathrm{~S}$ rRNA. Antimicrob Agents Chemother 45: 1-12, 2001.

13. Misyurina OY, Chipitsyna EV, Finashutina YP, Lazarev VN, Akopian TA, Savicheva AM and Govorun VM: Mutations in a 23S rRNA gene of Chlamydia trachomatis associated with resistance to macrolides. Antimicrob Agents Chemother 48: 1347-1349, 2004.

14. Binet R and Maurelli AT: Frequency of development and associated physiological cost of azithromycin resistance in Chlamydia psittaci $6 \mathrm{BC}$ and C. trachomatis L2. Antimicrob Agents Chemother 51: 4267-4275, 2007.

15. Shang S, Xia L, Shao C, et al: In vitro effects of various antibiotics alone and in combination with other antibiotics against Chlamydia trachomatis. Chin J Dermatol 38: 282-284, 2005.

16. Welsh LE, Gaydos CA and Quinn TC: In vitro evaluation of activities of azithromycin, erythromycin, and tetracycline against Chlamydia trachomatis and Chlamydia pneumoniae. Antimicrob Agents Chemother 36: 291-294, 1992.

17. Suchland RJ, Geisler WM and Stamm WE: Methodologies and cell lines used for antimicrobial susceptibility testing of Chlamydia spp. Antimicrob Agents Chemother 47: 636-642, 2003.

18. Clinical and Laboratory Standards Institute (CLSI): Performance Standards for Antimicrobial Susceptibility Testing; Nineteenth Informational Supplement (M100-S19). CLSI, Wayne, PA, USA, 2009.

19. Michéa-Hamzehpour M, Kahr A and Pechère JC: In vitro stepwise selection of resistance to quinolones, beta-lactams and amikacin in nosocomial gram-negative bacilli. Infection 22 (Suppl 2): S105-S110, 1994. 